PROCEEDINGS OF THE AMERICAN MATHEMATICAL SOCIETY

Volume 124, Number 10, October 1996

\title{
GENERALIZED ALUTHGE TRANSFORMATION ON $p$-HYPONORMAL OPERATORS
}

\author{
TAKAYUKI FURUTA \\ (Communicated by Palle E. T. Jorgensen) \\ Dedicated to Professor C. R. Putnam on his retirement \\ with respect and affection
}

\begin{abstract}
We shall introduce a generalized Aluthge transformation on $p$ hyponormal operators and also, by using the Furuta inequality, we shall give several properties on this generalized Aluthge transformation as further extensions of some results of Aluthge.
\end{abstract}

\section{INTRODUCTION}

An operator means a bounded linear operator on a Hilbert space. It is well known that an operator $T$ can be decomposed into $T=U|T|$ where $U$ is a partial isometry with $N(U)=N(|T|)$, where $N(X)$ denotes the kernel of an operator $X$ and $T=U|T|$ is said to be the polar decomposition of an operator $T$ if this kernel condition $N(U)=N(|T|)$ is satisfied.

An operator $T$ is said to be hyponormal if $T^{*} T \geq T T^{*}$ and also $T$ is said to be semihyponormal if $\left(T^{*} T\right)^{1 / 2} \geq\left(T T^{*}\right)^{1 / 2}$ and semihyponormal was introduced by $\mathrm{Xia}$ [12]. It is known in Xia [12] that there exists an example which is semihyponormal but not hyponormal, that is, the class of semihyponormal operators properly contains the one of hyponormal operators. An operator $T$ on a Hilbert space $H$ is said to be p-hyponormal if

$$
\left(T^{*} T\right)^{p} \geq\left(T T^{*}\right)^{p} \quad \text { for a positive number } p .
$$

The class of $p$-hyponormal has been defined as an extension of semihyponormal and also it has been studied by many authors, mainly Aluthge [1], [2], Duggal [3] and Xia [13].

For a $p$-hyponormal operator $T=U|T|$, Aluthge [1] introduced the operator $\widetilde{T}=|T|^{1 / 2} U|T|^{1 / 2}$ which is called Aluthge transformation and Aluthge [1] showed very interesting results on $\widetilde{T}$. As an extension of $\widetilde{T}=|T|^{1 / 2} U|T|^{1 / 2}$, we shall consider $\widetilde{T}=|T|^{q} U|T|^{q}$ for a positive number $q$ which is not necessarily $1 / 2$ and by using the Furuta inequality, we shall give several properties on $\widetilde{T}=|T|^{q} U|T|^{q}$ for a positive number $q$, which can be considered as further extensions of some results in Aluthge [1] and [2].

Received by the editors March 9, 1995.

1991 Mathematics Subject Classification. Primary 47B20, 47A63.

Key words and phrases. p-hyponormal operator, Aluthge transformation, Furuta inequality.

The author's research was supported in part by Grant-in-Aid for Scientific Research. 
Our main result is as follows: let $T=U|T|$ be the polar decomposition of $p$-hyponormal for $1 \geq p>0$ with $N(T)=N\left(T^{*}\right)$. Then $\widetilde{T}=|T|^{q} U|T|^{q}$ is $\frac{1}{2}\left(1+\frac{p}{q}\right)$ hyponormal for any $q$ such that $q \geq p$. This result implies the following one by Aluthge [1]: Let $T=U|T|$ be $p$-hyponormal for $0<p<\frac{1}{2}$ and $U$ be unitary. Then $\widetilde{T}=|T|^{1 / 2} U|T|^{1 / 2}$ is $\left(p+\frac{1}{2}\right)$-hyponormal.

Let $T=U|T|$ be the polar decomposition of $p$-hyponormal for $1 \geq p>0$. Then $\widetilde{T}=|T|^{q} U|T|^{q}$ is hyponormal for any $q$ such that $p \geq q>0$.

Also we have the following corollary: Let $T=U|T|$ be the polar decomposition of an invertible $p$-hyponormal operator for $1 \geq p>0$, where $U$ is a unitary operator and $|T|>0$. Let $q$ and $r$ be any positive numbers such that $q \geq p$ and $\frac{1}{2}\left(1+\frac{p}{q}\right) \geq r$. Also let $\widetilde{T}=\widetilde{U}|\widetilde{T}|$ be the polar decomposition of an operator $\widetilde{T}=|T|^{q} U|T|^{q}$. Then $\widetilde{\widetilde{T}}=|\widetilde{T}|^{r} \widetilde{U}|\widetilde{T}|^{r}$ is hyponormal. Also this corollary can be considered as an extension of Corollary 3.3 in Aluthge [2].

\section{Statement of the Results}

Theorem 1. Let $T=U|T|$ be the polar decomposition of $p$-hyponormal for $1 \geq$ $p>0$ with $N(T)=N\left(T^{*}\right)$. Then $\widetilde{T}=|T|^{q} U|T|^{q}$ is $\frac{1}{2}\left(1+\frac{p}{q}\right)$-hyponormal for any $q$ such that $q \geq p$.

Remark 1. $\widetilde{T}=|T|^{q} U|T|^{q}$ in Theorem 1 turns out to be semihyponormal because $1 \geq \frac{1}{2}\left(1+\frac{p}{q}\right)>\frac{1}{2}$ holds for $1 \geq p>0$ and $q \geq p$ and we have only to apply the Löwner-Heinz theorem to this inequality.

We need the following result to give a proof of Theorem 1 .

Theorem A (Furuta inequality [6]). If $A \geq B \geq 0$, then for each $r \geq 0$

$$
\left(B^{r} A^{p} B^{r}\right)^{1 / q} \geq\left(B^{r} B^{p} B^{r}\right)^{1 / q}
$$

and

$$
\left(A^{r} A^{p} A^{r}\right)^{1 / q} \geq\left(A^{r} B^{p} A^{r}\right)^{1 / q}
$$

hold for each $p$ and $q$ such that $p \geq 0, q \geq 1$ and $(1+2 r) q \geq p+2 r$.

We remark that Theorem A yields the Löwner-Heinz theorem [9], [11] when we put $r=0$ in (i) or (ii) stated above: if $A \geq B \geq 0$ ensures $A^{\alpha} \geq B^{\alpha}$ for any $\alpha \in[0,1]$. Alternative proofs of Theorem A are given in [4], [7], [10] and an elementary proof is shown in [8].

Proof of Theorem 1. We have only to employ the ingenious proof of Theorem 2 in Aluthge [1] based on Theorem A. Firstly we recall that $U^{*} U|T|^{q}=|T|^{q}$ holds for any $q>0$ since $U^{*} U$ is the initial projection onto $\overline{R(|T|)}$, so that $T$ is $p$-hyponormal for $p>0$ is equivalent to the following

$$
|T|^{2 p} \geq U|T|^{2 p} U^{*} \text { holds for } p>0 .
$$

By (1) we have

$$
U^{*}|T|^{2 p} U \geq U^{*} U|T|^{2 p} U^{*} U=|T|^{2 p} \text { for any } p>0 .
$$

By (1) and (2), we have

$$
U^{*}|T|^{2 p} U \geq|T|^{2 p} \geq U|T|^{2 p} U^{*} \text { for any } p>0 .
$$


Next we shall show the following equation for any $r>0$

$$
\left(U^{*}|T| U\right)^{r}=U^{*}|T|^{r} U \text { holds under the hypothesis } N(T)=N\left(T^{*}\right) .
$$

The hypothesis $N(T)=N\left(T^{*}\right)$ is equivalent to $N(T)^{\perp}=N\left(T^{*}\right)^{\perp}$ and also this means that the initial projection coincides with the final projection, that is, $U U^{*}=$ $U^{*} U$. Therefore we have

$$
\begin{aligned}
\left(U^{*}|T| U\right)^{2} & =U^{*}|T| U U^{*}|T| U \\
& =U^{*}|T| U^{*} U|T| U^{*} \quad \text { by } U U^{*}=U^{*} U \\
& =U^{*}|T|^{2} U \text { since } U^{*} U \text { is the initial projection }
\end{aligned}
$$

and similarly $\left(U^{*}|T| U\right)^{n / m}=U^{*}|T|^{n / m} U$ holds by induction for any natural number $n$ and $m$, so that the continuity of an operator yields $\left(U^{*}|T| U\right)^{r}=U^{*}|T|^{r} U$ by attending $n / m \rightarrow r$, so we have (4).

Let $A=U^{*}|T|^{2 p} U, B=|T|^{2 p}$ and $C=U|T|^{2 p} U^{*}$. Then for any $p>0$ and $q>0$

$$
A^{2 q / 2 p}=\left(U^{*}|T|^{2 p} U\right)^{2 q / 2 p}=\left(U^{*}|T|^{2 q} U\right) \text { holds by }(4)
$$

and also $C^{2 q / 2 p}=\left(U|T|^{2 p} U^{*}\right)^{2 q / 2 p}=C|T|^{2 q} U^{*}$ holds in general.

Applying Theorem A to (3) since $\left(1+2 \frac{q}{2 p}\right) \frac{2 q}{p+q}=\frac{2 q}{2 p}+2 \frac{q}{2 p}$ and $\frac{2 q}{p+q} \geq 1$ for $1 \geq p>0$ and $q \geq p$, we get

$$
\begin{aligned}
\left(\widetilde{T}^{*} \widetilde{T}\right)^{(p+q) / 2 q} & =\left(|T|^{q} U^{*}|T|^{2 q} U|T|^{q}\right)^{(p+q) / 2 q} \\
& =\left(B^{q / 2 p} A^{2 q / 2 p} B^{q / 2 p}\right)^{(p+q) / 2 q} \\
& \geq B^{(2 q / 2 p+2 q / 2 p)((p+q) / 2 q)}=B^{1+q / p}
\end{aligned}
$$

and

$$
\begin{aligned}
\left(\widetilde{T} \widetilde{T}^{*}\right)^{(p+q) / 2 q} & =\left(|T|^{q} U|T|^{2 q} U^{*}|T|^{q}\right)^{(p+q) / 2 q} \\
& =\left(B^{q / 2 p} C^{2 q / 2 p} B^{q / 2 p}\right)^{(p+q) / 2 q} \\
& \leq B^{(2 q / 2 p+2 q / 2 p)((p+q) / 2 q)}=B^{1+q / p}
\end{aligned}
$$

Hence $\left(\widetilde{T}^{*} \widetilde{T}\right)^{(p+q) / 2 q} \geq\left(\widetilde{T} \widetilde{T}^{*}\right)^{(p+q) / 2 q}$, that is, $\widetilde{T}$ is $\frac{1}{2}\left(1+\frac{p}{q}\right)$-hyponormal.

Theorem 2. Let $T=U|T|$ be the polar decomposition of p-hyponormal for $1 \geq$ $p>0$. Then $\widetilde{T}=|T|^{q} U|T|^{q}$ is hyponormal for any $q$ such that $p \geq q>0$.

Proof. As $T$ is $p$-hyponormal for $p>0, T$ is $q$-hyponormal for $q$ such that $p \geq q>0$ by the Löwner-Heinz theorem, then by (3) we have

$$
U^{*}|T|^{2 q} U \geq|T|^{2 q} \geq U|T|^{2 q} U^{*} \text { for any } q \text { such that } p \geq q>0,
$$

therefore (5) implies

$$
\left(\widetilde{T}^{*} \widetilde{T}\right)-\left(\widetilde{T} \widetilde{T}^{*}\right)=|T|^{q}\left(U^{*}|T|^{2 q} U-U|T|^{2 q} U^{*}\right)|T|^{q} \geq 0
$$

for any $q$ such that $p \geq q>0$, that is, $\widetilde{T}$ is hyponormal, so the proof is complete.

Corollary 3. Let $T=U|T|$ be the polar decomposition of an invertible p-hyponormal operator for $1 \geq p>0$, where $U$ is a unitary operator and $|T|>0$. Let $q$ and $r$ be any positive numbers such that $q \geq p$ and $\frac{1}{2}\left(1+\frac{p}{q}\right) \geq r$. Also let $\widetilde{T}=\widetilde{U}|\widetilde{T}|$ be the polar decomposition of an operator $\widetilde{T}=|T|^{q} U|T|^{q}$. Then $\widetilde{\widetilde{T}}=|\widetilde{T}|^{r} \widetilde{U}|\widetilde{T}|^{r}$ is hyponormal. 
Proof. As $T$ is invertible $p$-hyponormal, $1 \geq p>0, T$ can be decomposed into $T=U|T|$, where $U$ is unitary and $|T|>0$, so that Theorem 1 ensures that $\widetilde{T}=$ $|T|^{q} U|T|^{q}$ is $\frac{1}{2}\left(1+\frac{p}{q}\right)$-hyponormal for $q$ such that $q \geq p$. Then Theorem 2 yields that $\widetilde{\widetilde{T}}=|\widetilde{T}|^{r} \widetilde{U}|\widetilde{T}|^{r}$ is hyponormal for any $r$ such that $\frac{1}{2}\left(1+\frac{p}{q}\right) \geq r$, so the proof is complete.

We remark that our Theorem 1 yields the following Theorem B in Aluthge [1] because $U$ in Theorem 1 can be extended to become unitary under the hypothesis $N(T)=N\left(T^{*}\right)$ by Theorem 4 in Furuta [5].

Theorem B (Aluthge [1]). Let $T=U|T|$ be p-hyponormal for $0<p<\frac{1}{2}$ and $U$ be unitary. Then $\widetilde{T}=|T|^{1 / 2} U|T|^{1 / 2}$ is $\left(p+\frac{1}{2}\right)$-hyponormal.

Also we remark that Corollary 3 can be considered as an extension of Corollary 3.3 in Aluthge [2].

\section{ADDENDUM}

We have the following slight extension of Theorem 1 by slightly modifying the proof of Theorem 1.

Theorem $1^{\prime}$. Let $T=U|T|$ be the polar decomposition of p-hyponormal for $1 \geq$ $p>0$ with $N(T)=N\left(T^{*}\right)$. Then $\widetilde{T}=|T|^{s} U|T|^{t}$ is $\left(\frac{p+s}{s+t}\right)$-hyponormal for any $s \geq 0$ and $t \geq \operatorname{Max}\{p, s\}$.

Professor A. Aluthge has kindly sent his excellent preprint in which one of them is overlapped with ours.

After reading our preprint, Professor T. Huruya kindly pointed out that $N(T)=$ $N\left(T^{*}\right)$ in Theorem 1 is unnecessary and his proof is quite ingenious based on Xia's lemma.

\section{REFERENCES}

1. A. Aluthge, On p-hyponormal operators for $0<p<1$, Integral Equations Operator Theory 13 (1990), 307-315. MR 91a:47025

2. $\_$Properties of p-hyponormal operators, unpublished dissertation, Vanderbilt University, Nashville, TN, August 1990.

3. B. P. Duggal, On p-hyponormal contraction, Proc. Amer. Math. Soc. 123 (1995), 81-86. MR 95d: 47025

4. M. Fujii, Furuta's inequality and its mean theoretic approach, J. Operator Theory 23 (1990), 67-72. MR 91g:47012

5. T. Furuta, On the polar decomposition of an operator, Acta Sci. Math. (Szeged) 46 (1983), 261-268. MR 86d:47027

6.,$A \geq B \geq 0$ assures $\left(B^{r} A^{p} B^{r}\right)^{1 / q} \geq B^{(p+2 r) / q}$ for $r \geq 0, p \geq 0, q \geq 1$ with $(1+2 r) q \geq p+2 r$, Proc. Amer. Math. Soc. 101 (1987), 85-88. MR 89b:47028

7. - A proof via operator means of an order preserving inequality, Linear Algebra Appl. 113 (1989), 129-130. MR 89k:47023

8. $\_$, Elementary proof of an order preserving inequality, Proc. Japan Acad. 65 (1989), 126. MR 90g:47029

9. E. Heinz, Beiträge zur Störungstheorie der Spektralzerlegung, Math. Ann. 123 (1951), 415438. MR 13:471f

10. E. Kamei, A satellite to Furuta's inequality, Math. Japon. 33 (1988), 883-886. MR 89m:47011

11. K. Löwner, Über monotone Matrixfunktionen, Math. Z. 38 (1934), 177-216. 
12. D. Xia, On the non-normal operators - semihyponormal operators, Sci. Sinica 23 (1980), 700713. MR 82g: 47020

13. - Spectral theory of hyponormal operators, Birkhäuser Verlag, Boston, 1983. MR $\mathbf{8 7 \mathbf { j }}: 47036$

Department of Applied Mathematics, Faculty of Science, Science University of Tokyo, 1-3 Kagurazaka, Shinjuku, Tokyo 162, Japan 